\title{
Mechanical Properties of Weft Knitted Fabrics in Fully Stretched Status along Courses Direction: Geometrical Model Aspect
}

\author{
Dariush Semnani \\ Department of Textile Engineering, Isfahan University of Technology, Isfahan, 84156-83111 Iran \\ *Corresponding Author: d_semnani@cc.iut.ac.ir
}

Copyright (c) 2013 Horizon Research Publishing All rights reserved.

\begin{abstract}
Weft knitted fabric structures have got new application in the modern technical textile such as seat belt and packaging fabrics. The weft knitted fabric is applied for technical textiles commonly as composites to resist against tension along courses direction in jamming status. In this study, mechanical properties of weft knitted fabrics with different structures of single jersey and double jersey in fully stretched status of jamming were considered by geometrical modeling. Ultra high tenacity nylon 6.6 yarn was used in manufacturing of weft knitted fabrics. Consequently the tenacity of the sample fabrics is assessed by a tensile tester. Mechanical properties of the fabrics are compared with theoretical modeling. The results show that the weft knitted fabrics with Rib structure have a superior tenacity. Also, double jersey fabrics of Full Cardigan and Full Milano represent better mechanical properties in comparison with single jersey fabrics.
\end{abstract}

Keywords weft knitted fabrics; geometrical modeling; mechanical properties; jamming status, knitted stitch

\section{Introduction}

Textile products of technical applications have been developed in protection and safety. The most important merit in those applications is high strength and work of rapture properties of knitted fabric. The belts of packaging and protection applications must present high strength with high work up to rapture property [1].

Many researches have been concerned in design and study on mechanical properties of fabric composites with reinforcement polymers. Most of composites are produced with compositions of woven fabrics of polyester, nylon 6 , nylon 6.6 and tenser yarns and reinforcement polymers as heat treated binders.

Although most of those composites have remarkable strength, but, none of them have enough elongation property. Therefore, the work up to rapture is not sufficient for packaging applications [1,2 and 3].

Other researches have been done to develop composite industry for simple knitted fabrics as base fabric in composites with shrinkable polymers of heat treated binders. These products presented better mechanical properties specially, regarding to impact resistance [4 and 5]. In these researches the effect of fabric structure has not been considered, sufficiently. Many researches have been done to consider stress/strain properties of knitted composites to make strong materials applicable in heavy industries $[6,7$ and 8]. Also impact resistance has been investigated in knitted composites to achieve high resistant materials for heavy lift applications [9, 10,11 and 12].

Weft knitted fabrics is made from connecting of yarn loops in hieratical courses. Each loop is consisted of one head loop, two vertical stems and two horizontal half circles as lower stems. While vertical force is applied along courses to the fabric, the loop elongated in vertical position and jamming phenomena is occurred in vertical position. This matter is occurred in horizontal position while the force is applied along walls. Therefore, the knitted fabrics have remarkable work up to rapture resistance against both vertical and horizontal forces specially, forces along courses. Weft knitted fabrics which are knitted in one needle series have hieratical courses in one surface. The other rib fabrics which are knitted in two needle series (cylinder/dial in circular and two beds in flat knitting machines) have two series of courses in two surfaces. In these kinds of knitted fabrics the loops are connected together in two directions of both courses. Therefore, it seems rib fabrics can present better mechanical properties in comparison with plain ones. Elements of knitted fabrics, which are knit, tuck and skip stitches, demonstrate different structures of fabric depend on design of fabric. Usually, tuck and skip stitches cause more stability and less shrinkage in knitted fabric. Skip stitches have no noticeable effect on thickness of fabric while tuck stitches causes increasing thickness and covering factor of fabric [13]. 
Popper has introduced the fully stretched geometrical modeling as jamming status of knitted fabric for both vertical and horizontal stretched positions. That of geometrical model is applied for plain fabric of single jersey just in the simple jamming status [14]. Other researchers developed Popper's assumptions by energy modeling method in stretched plain fabric too [15]. The aim of this research is investigation of mechanical behavior of different knitted fabrics in both kinds of plain and rib with various structures using knit, tuck and skip stitches to find suitable fabric with super high strength properties which could be applicable in industrial belts. Although finite element models can be used to consider the mechanical behavior model of this kinds of fabrics in jamming situation but these models are limited to yarn parameters which are very affected by yarn regularity. In textile, the yarns are not completely regular. Also, the structure of knitted fabric is deformed according to yarn elongation. Therefore, the simple geometrical model has been selected to compare various structures of knitted belts.

\section{Material and Methods}

\subsection{Geometrical Modeling in jamming status}

The mechanical behavior of knitted fabric is not a linear model of mechanical behavior. The knitted fabric has various behaviors before fully stretched and jamming status. In the starting point of stretching, the tension causes more strain with low tension while, the fabric has more strength against stretching tension in jamming status. The structure of fabric is most important matter while, yarn type and count is the same for every fabric.

The knitted structures are caparisoned together based on their dimensional stitches and knit elements which are structural component of fabric. In various fabrics, different elements of knit, tuck and skip stitches are arranged to make structure of fabric. The jamming status of knitted elements in simple form of plain knit fabric has been shown by previous researches. The maximum tension is acted on each parts of knitted structure in jamming status. In jamming status, stitches deformed as straight direction of acting tension. The rapture of structure is depended on acting tension on each part of knitted structure element. If the acting tension on each element is low the maximum tension could be increased up to rapture of parts of elements [14 and 15].

Whereas the knit elements rotate in wale direction and the yarn diameter is ignorable against length of loop, the assumption of straight parts in jammed element seems true. In this situation, the tension acts on elements approximately uniform and acting tension on each knit element is named " $\partial T "$.

There are two phenomena in the jamming status. One of them is compression of knit loop head and sinker loop as presented in figure 1a. The other is rupturing tension which is occurred on both stems of knit loop as presented in figure $1 \mathrm{~b}$. The important matter is finding critical phenomena which are more effective on rupturing occurrence.

The compression occurrence is considered in knit and sinker loops. In jamming status the stems of knit loop are straight in vertical position along course. Therefore, the head of knit loop is in contact with two sinker loops as Figure 1a. By more tension one of sinker loops is moved under the other sinker loop because of slippage occurrence as figure $1 \mathrm{~b}$ as full jamming status. If the total tension on each element is named " $T$ " in (N) in the head loop part the tension is calculated from equation 1 where, the contact part is half of circle. The total tension on each element can be defined to compression tension between loops $T_{c}$ and stretching tension on stems of stitches $T_{S}$

$$
T=\frac{\pi d y}{4} \int_{-\pi / 4}^{\pi / 4} \partial T_{c} \cdot d \cdot d \theta
$$

Where $\partial T_{c}, d_{y}$ and $\theta$ are partial tension of compression form on loop, yarn diameter and angle of stitch, respectively. $\partial T$ is found from equation 1 as equation 2 .

$$
\partial T_{c}=\frac{8 T}{\pi^{2} d_{y}^{2}}
$$

In jamming status the tension acting on stems of loops is not same as compression tension of knit loop head and sinker loop. The tension on stems named " $\partial T_{s}$ "is stretching tension can be defined as equation 3 .

$$
\partial T_{S}=\frac{4 T}{\pi d_{y}^{2}}
$$

By primary comparison between " $\partial T_{S}$ " and " $\partial T_{\mathcal{C}}$ ", it seems that " $\partial T_{C}=0.637 \partial T_{S}$ ". Therefore, the compression tension is less than stretching tension and the rupturing probability is very high in stems. It means that the model of comparison between different structures must be presented based on stretching tension on loop stems. Thus, the yarn as Viscoelastic material which has a property between elastic and plastic materials can resist easily against compression tension by deformation and transferring the compression tension to stretching tension on stems. This matter can be assumed as second reason of stretching tension importance against compression tension of knit loop head and sinker loop. The third reason is the different length of filaments in stems which causes increasing rate of rupturing against stretching tension. Therefore, in the geometrical model of jamming status compression tension is not comprised in different structures and just stretching tension is comprised.

The rib structure has two knit stitch which are located behinds together. There is one stem between both of them for connection of stitches together. The partial tension " $\partial T$ " is occurred in both of them, therefore there is " $\partial T / 2$ " on each stitch as figure $2 \mathrm{a}$ in jamming status. Each stitch has two complete loop sides in direction of tension and one 
connection stem. The acting tension on each part of rib element is " $\partial T / 5$ ".

In plain structure of figure $2 b$, there is one stitch which is consisted two loop sides. In this structure, acting tension on each part of element is " $\partial T / 2$ " for jamming status. Therefore it is expected that the strength of plain structure will be three times less than rib structure. In skip structure of figure $2 \mathrm{c}$, usually there are one skip stitch and one knit stitch.

The skip stitch has one stem part and knit structure have two loop sides. Therefore, the acting tension in each element is $\partial T / 3$ as jamming situation. This matter is different for tuck structure of figure $2 \mathrm{~d}$ where, tuck stitch presents two stems. In this structure, knit stitch has two loop sides and tuck has two stems. Therefore, acting tension for each part of tuck structure element is $\partial T / 4$ while jamming is occurred.

The mentioned models presents that, rib structure has less acting tension on each part of that's element in comparison with other structures. Therefore, it is expected that rib structure presents more strength up to rapture. Tuck and skip structures respectively, have better resistance against acting tension because of number of element parts in those of structures.

The plain structure has two parts in that of element and therefore can present low resistance against tension. In yarn fold just one part is resistance element against tension. The model suggests that it is possible to achieve more strength in belt based on the number of resistance parts in knit element by knitting structures. To investigate reality of this simple model some experiments were done on fabric samples of various knit structures.

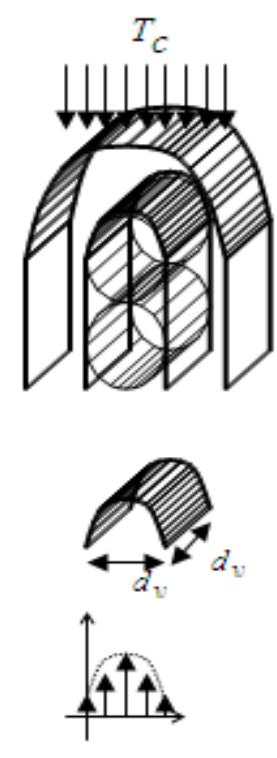

Compressiontension

(a)
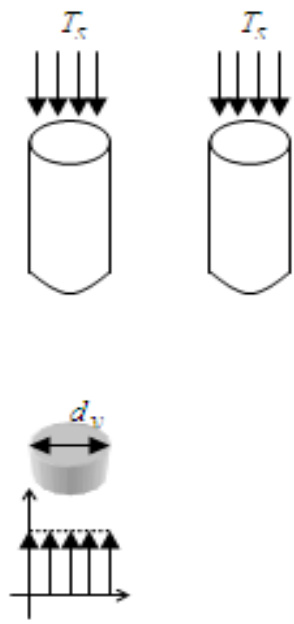

Stretching tension

(b)
Figure 1. Analysis of surface tension (a: compression tension on knit loop head and sinker loop and b: Stretching tension on loop stems)

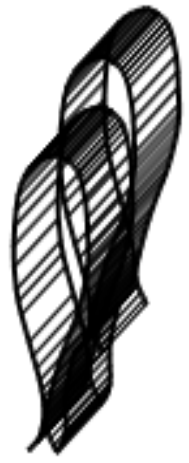

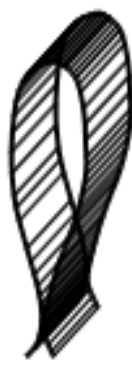

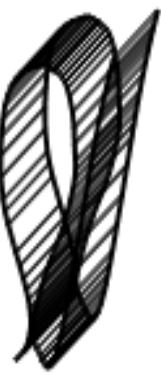

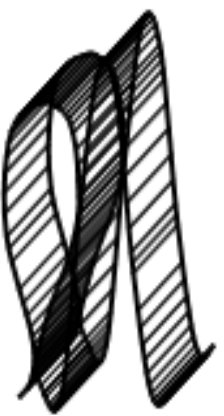

d
Figure 2. Schematic of knit structures in shape of profile (a: rib element, b: plain element, c: skip element and d: tuck element)

\subsection{Experiments}

Regarding to theoretical modeling, it is assumed that various fabric structures demonstrate different mechanical properties. This matter is base of sample preparation and plan of experiments. The knitted fabrics were knitted in one flat knitting machine with gauge of 5 (needles/inch) from high tenacity yarn of nylon 6.6 filaments $(900 \mathrm{den})$ with strength ( $\mathrm{F} \max ) 55.27 \mathrm{~N}$, elongation ( $\mathrm{L}$ at $\mathrm{F} \max ) 67.25$ $\mathrm{mm}$, strain $26.90 \%$ and work up to rapture $2.09 \mathrm{Nm}$ as raw material (produced in ZTC company). Whole knitted fabrics were knitted in 10 walls which is approximately equal to width of common industrial packaging belts.

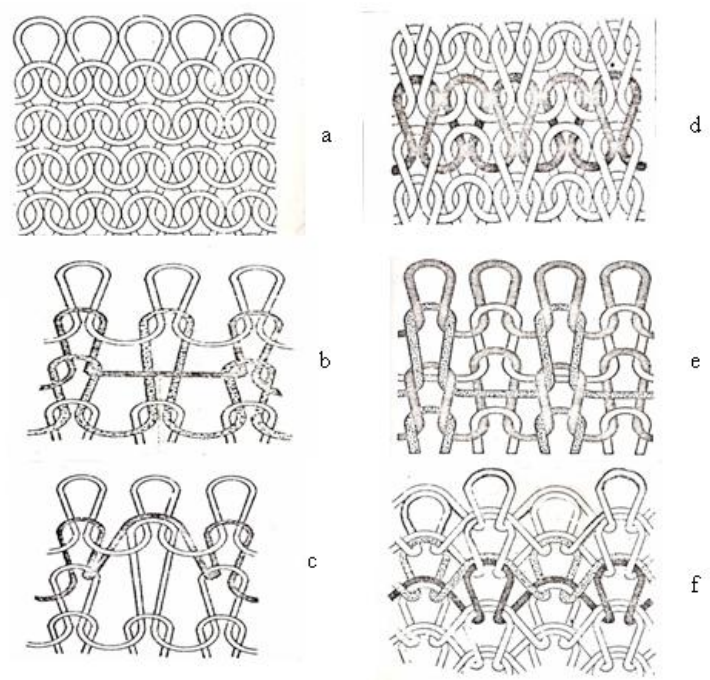

Figure 3. Structures of fabric samples a)Plain, b)Cross Miss, c)Single Cross Tuck, d)Rib 1x1, e)Full Milano and f)Full cardigan fabrics

To investigate effect of fabric structure on its mechanical behavior and also to determine suitable structure of fabric which presents high strength and work up to rapture, different types of fabrics were prepared from the yarn sample. Regarding to theoretical assumptions, knit, tuck and skip stitches causes different variations in mechanical 
properties of knitted fabric. Therefore, different types of fabric samples were prepared from those elements as fabric structure. The samples are: a)Plain, b)Cross Miss, c)Single Cross Tuck, d)Rib 1x1, e)Full Milano and f)Full cardigan. The structures of fabric samples have been demonstrated in figure 3. Thirty fabric samples from each structure were knitted in a flat weft knitting machine with gauge of 5 needles per centimeter. The "ZWICK" tensile apparatus was used to measure mechanical properties of the textile samples. This apparatus works based on the constant rate of elongation procedure. First of all we produced many samples from high tenacity nylon yarns with different designs. Then we measured and compared their mechanical properties.

\section{Results and Discussion}

All of samples of fabrics were experimented by "ZWICK" apparatus for investigation of mechanical properties and comparison with theoretical assumptions. The results of experiments have been arranged in Table 1.

Table 1. Breaking load properties results

\begin{tabular}{ccccc}
\hline & \multicolumn{4}{c}{ Breaking Load (N) } \\
\cline { 2 - 5 } Fabric Sample & Mean & $\begin{array}{c}\text { Standard } \\
\text { Deviation }\end{array}$ & Max & Min \\
\cline { 2 - 5 } & 285.72 & 7.88 & 301.53 & 272.10 \\
Plain & 272.72 & 7.73 & 287.00 & 258.92 \\
Cross miss & 277.48 & 8.32 & 298.00 & 263.24 \\
Single cross tuck & 610.91 & 18.07 & 653.00 & 579.65 \\
Rib 1-1 & 490.45 & 13.41 & 516.32 & 465.92 \\
Full milano & 481.91 & 12.85 & 507.77 & 458.21 \\
Full cardigan & & & &
\end{tabular}

Force at rapture or specific strain shows resistance of fiber against the gradual, continuous and uniform rate of force. Whereas, breaking load is stretching load based on theoretical modeling, the load is the final resisted load by loop stems in jamming status. Therefore, strain of fabric before jamming status and work of rupturing from non stretched fabric is not important. The strain of fabric samples after jamming status was very small. This matter presents that the work of rapture from jamming status to fully ruptured fabric just is dependent on strength of stems against breaking load which is stretching tension on stems. From geometrical models of different fabric structures, it is expected that Rib 1-1 may has most strength where, full cardigan and full milano structures are other strong structures, respectively. Also, it was expected that single cross tuck and cross miss structures are weaker than plain structure. This matter can be seen for stretching and compression tensions too.

The results presented in table 2 demonstrates that Rib structure can resist against stretching force up to 7.6364 Gpa but compression tension is $4.8616 \mathrm{Gpa}$. These results are similar for other structures and compression tension is less than stretching tension. Although double face knits provide more stretching resistance because they have twice more resisting elements as stems of loops but the compression tension leads to damaging the cross section of fibers and rupturing mechanisms would be happened. Visual observations presents that rupturing of stitches have been happened in head of loop which is result of compression tension mechanism.

Table 2. Tension results in stretching and compressing

\begin{tabular}{ccc}
\hline \multirow{2}{*}{ Fabric Sample } & \multicolumn{2}{c}{ Breaking Tension (Gpa) } \\
\cline { 2 - 3 } & $\partial T_{c}$ & $\partial T_{s}$ \\
\hline Plain & 2.2738 & 3.5715 \\
Cross miss & 2.1703 & 3.4090 \\
Single cross tuck & 2.2082 & 3.4685 \\
Rib 1-1 & 4.8616 & 7.6364 \\
Full milano & 3.9030 & 6.1306 \\
Full cardigan & 3.8350 & 6.0239 \\
\hline
\end{tabular}

The result of experiments which is presented as figure 4 presents that Rib 1-1 structure is the strongest among the others which is conformed with geometrical model too. Also, it is seen that among the one- face and two-face cylinder fabrics the designs with the most knit are stronger than the others and this means that tuck and skip decrease the strength in vertical direction. The reason of this phenomena is that tuck and skip stitches which have less take part in vertical strength where, because of their position in fabric as drawing horizontal direction, the strength in horizontal direction is more than vertical direction.

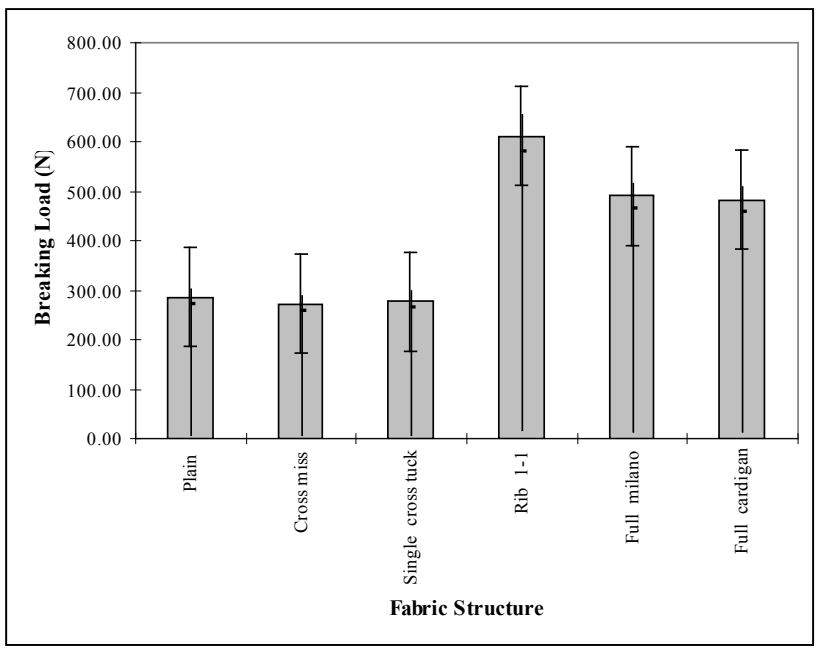

Figure 4. Comparison between breaking load of different fabric structures (Test for 30 samples of each structure)

The double cylinder fabrics are stronger than the single one haw ever it is because of doubling the thickness of fabrics. The strength increasing rate is not linear which is not same as expectations of geometrical model. The non linear behavior of fabric strength difference between double 
and single fabrics may be occurred because of slippage of the both courses of double face fabric in jamming status.

It seems that strength of yarn decreased if its form a loop, but the greater strength of two-face cylinder fabrics is the more thickness of fabrics or doubling the amount of yarns. The cross miss has the least longitudinal strength because the least of skip. The results show that Rib1-1 is the best choice among the others by former mentioned reasons. This matter is conformed by geometrical modeling too. Full-milano sample is stronger than Full-cardigan sample despite of geometrical modeling aspect. The reason of more strength in Full milano in comparison with Full cardigan structure may be explained as further number of knit stitch in full-milano sample in comparison with full cardigan even full milano has not tuck stitches.

Elongation at rapture shows the amount or percent of length increased when the sample is broken .Work of rupture shows the amount of required energy to rapture the sample. We must specify that which of these three parameters is important with respect to the aim of the work. From table 3 and figure 5, it can be seen that elongation and work of rapture of Rib 1.1 are the optimum ones among the others. We can also see that among the one- face and two-face cylinder fabrics the designs with the most knit are better than the others and this means that tuck and skip decrease the strength in vertical direction. The reason of this phenomena is that tuck and skip stitches which have less take part in vertical strength where, because of their position in fabric as drawing horizontal direction, the strength in horizontal direction is more than vertical direction

Table 3. Mechanical properties results

\begin{tabular}{cccc}
\hline Fabric Sample & $\begin{array}{c}\text { F } \max \\
(\mathrm{N})\end{array}$ & $\begin{array}{c}\text { Strain } \\
(\%)\end{array}$ & $\begin{array}{c}\text { Work of } \\
\text { rupture }(\mathrm{Nm})\end{array}$ \\
\hline Plain & 273.19 & 16.79 & 2.80 \\
Cross miss & 259.96 & 13.01 & 2.25 \\
Single cross tuck & 264.30 & 16.52 & 2.76 \\
Rib 1-1 & 581.98 & 17.98 & 5.84 \\
Full milano & 467.79 & 11.95 & 4.01 \\
Full cardigan & 460.05 & 10.88 & 3.38 \\
10 yarn fold & 335.13 & 9.31 & 2.09 \\
\hline
\end{tabular}

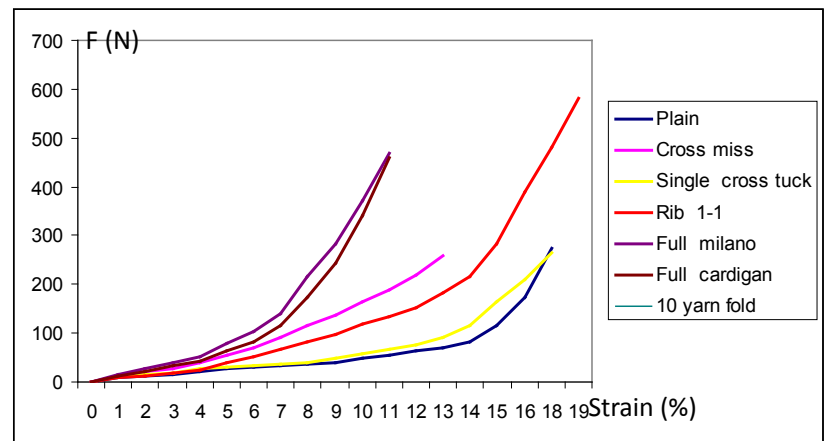

Figure 5. Stress-strain curvature of knitted fabric samples in course direction

\section{Conclusions}

The present work presented usage of ultra high tenacity nylon 6.6 yarns in manufacturing of weft knitted fabrics as ultra strength belts. The mechanical properties of the sample fabrics are assessed by a tensile tester. Tensile properties of the fabrics are compared with the geometrical modeling in jamming status. The results show that the weft knitted ultra high tenacity fabrics with rib structure have a superior tenacity.

The results showed that knit fabric with rib structure presents the most strength where Full milano and Full cardigan structures of knitted fabric have more strength after rib 1-1, respectively. Therefore, for packaging applications the rib structure is suitable. This could have economical advantages as well which in here would be a good alternative for the commercial seat belts and even packaging fabric. Various composites of knitted fabrics can be studied for better mechanical behavior in special applications too.

\section{REFERENCES}

[1] A. R. Horrocks, and S. Anand, " Handbook of technical textiles ", CRC Press, woodhead publishing, Cambridge, (2000).

[2] Z. A. Mohd Ishak, Y. W. Leong, M. Steeg, and J. Karger-Kocsis, "Mechanical properties of woven glass fabric reinforced in situ polymerized poly(butylene terephthalate) composites", Composites Science and Technology, 6(3-4), 390-398, (2007).

[3] P. Potluri, and V. S. Thammandra, "Influence of uniaxial and biaxial tension on meso-scale geometry and strain fields in a woven composite", Composite Structures, 77(3),405-418, (2007).

[4] S. Ramakrishna, "Characterization and modeling of the tensile properties of plain weft-knit fabric-reinforced composites", Composites Science and Technology, 57(1), p 1-22, 1997.

[5] S. Ramakrishna, N. K. Cuong, and H. Hmada, " Tensile properties of plain weft knitted glass fiber fabric reinforced epoxy composites", Journal of Reinforced Plastics and Composites, 16(10), p 946-966, (1997).

[6] An experimental investigation of the bearing strength of weft-knitted $1 \times 1$ rib glass fiber composites"Composite Structures, 78(3),392-396,(2007).

[7] B. Sun, H. Hu, and B. Gu, "Compressive behavior of multi-axial multi-layer warp knitted (MMWK) fabric composite at various strain rates", Composite Structures, 78(1), 84-90, (2007).

[8] M. Tercan, O. Asi, and A. Aktaş, "Determination of the critical crack length of notched weft-knitted glass fiber variable width composite plates",Composite Structures, 77(1), 111-119,(2007).

[9] Z. M. Huang, S. Ramakrishna, and A. A. O. Tay, "Modeling the stress/strain behavior of a knitted fabric-reinforced elastomer composite", Composites Science and Technology, 
60(5), 671-691, (2000).

[10] K. B. Cheng, K. C. Lee, T. H. Ueng, and K. J. Mou, "Electrical and impact properties of the hybrid knitted inlaid fabric reinforced polypropylene composites", Composites Part A: Applied Science and Manufacturing, 33(9), 1219-1226, (2002).

[11] O. A. Khondker, K. H. Leong, I. Herszberg, and H. Hamada, "Impact and compression-after-impact performance of weft-knitted glass textile composites", Composites Part A: Applied Science and Manufacturing, 36(5), 638-648, (2005).

[12] B. Gommers, I. Verpoest, and P. Houtte, "Analysis of knitted fabric reinforced composites: Part II. Stiffness and strength", Composites Part A: Applied Science and Manufacturing, 29(12), 1589-1601, (1998).

[13] J. F. Knapton, "Geometry of complex knitted structures", Textile Research Journal, 39(9), 889-892, (1969).

[14] P. Popper, "The theoretical behavior of a knitted fabric subjected to biaxial stresses", Textile Research Journal, 36, $148-157,(1966)$.

[15] B. M. MacRory, J. R. McCraith, and A. B. McNamara, " Biaxial load-extension properties of plain weft knitted fabric: A theoretical analysis:", Textile Research Journal, 45(10), p 746-760, (1975). 